\title{
Probing Compositional Variation within Hybrid Nanostructures
}

\author{
Benjamin D. Yuhas, ${ }^{\dagger, \perp}$ Susan E. Habas, ${ }^{\ddagger, \perp}$ Sirine C. Fakra, ${ }^{\S}$ and Taleb Mokari ${ }^{\ddagger, *}$ \\ ${ }^{\dagger}$ Department of Chemistry, University of California, Berkeley, California 94720, ${ }^{\dagger}$ The Molecular Foundry, Lawrence Berkeley National Laboratory, and ${ }^{\S}$ Advanced Light \\ Source, Lawrence Berkeley National Laboratory, 1 Cyclotron Road, Berkeley, California $94720 .{ }^{\perp}$ These authors contributed equally to this work.
}

$\mathrm{P}$ latinum-based bimetallic alloys are highly desirable for their potential in magnetic storage, sensing, and catalysis, ${ }^{1-5}$ often displaying properties that are independent of their constituent materials. ${ }^{6}$ In addition to changes in the geometric distribution of the individual atoms, the unique structure and bonding in bimetallics can also lead to alterations in the electronic structure of the material. For example, PtNi alloys show enhanced activity for oxygen reduction relative to pure $\mathrm{Pt}^{7}$ as do similar alloys, including $\mathrm{Pt}_{3} \mathrm{Co}^{8}{ }^{8} \mathrm{How}-$ ever, bimetallic nanomaterials are not as well-understood outside of singlecrystalline studies or theoretical modeling, and the practical application of more complex nanostructures is further complicated by factors such as varied growth conditions, the presence of surface stabilizing agents, and the increased surface-to-volume ratio of nanoscale materials. Bimetallic nanoparticles have even been shown to undergo dynamic metal segregation in response to oxidizing or reducing environments. ${ }^{9}$

As nanostructured systems increase in complexity, the potential for structural and compositional variations with respect to the individual components rises dramatically. Hybrid nanostructures, the combination of two or more nanoscale materials to form a new heterostructure, are being actively pursued for a number of applications, ${ }^{10}$ including device integration and assembly, ${ }^{11-13}$ chemical and biological sensing, ${ }^{14,15}$ and photocatalysis. ${ }^{16-23}$ In particular, the integration of a metallic component with an optically active semiconductor, directly in solution, can lead to novel materials that exhibit new functionalities that result from the coupling between the individual components. For example, photocatalytic heterostructures can promote efficient charge separa-
ABSTRACT We present a detailed analysis of the structural and magnetic properties of solution-grown PtCo - CdS hybrid structures in comparison to similar free-standing PtCo alloy nanoparticles. X-ray absorption spectroscopy is utilized as a sensitive probe for identifying subtle differences in the structure of the hybrid materials. We found that the growth of bimetallic tips on a $\mathrm{CdS}$ nanorod substrate leads to a more complex nanoparticle structure composed of a PtCo alloy core and thin CoO shell. The core-shell architecture is an unexpected consequence of the different nanoparticle growth mechanism on the nanorod tip, as compared to free growth in solution. Magnetic measurements indicate that the $\mathrm{PtC}-\mathrm{CdS}$ hybrid structures are superparamagnetic despite the presence of a $\mathrm{C} 00$ shell. The use of $X$-ray spectroscopic techniques to detect minute differences in atomic structure and bonding in complex nanosystems makes it possible to better understand and predict catalytic or magnetic properties for nanoscale bimetallic hybrid materials.

KEYWORDS: hybrid nanostructures - bimetallic nanoparticles core-shell · magnetic characterization - X-ray absorption spectroscopy

tion across a metal-semiconductor junction, followed by catalytic reaction at the surface of the metal component.

The development of semiconductormetal hybrid nanostructures for magnetic and catalytic applications requires a thorough understanding of the metallic structure, which is difficult to predict for a complex system where nucleation and growth proceeds on the tip of a semiconductor. In a previous communication, we reported the selective growth of $\mathrm{Pt}, \mathrm{PtCo}$, and PtNi nanoparticles on the tips of $\mathrm{CdS}$ rods in solution. ${ }^{24}$ Here, we present a detailed analysis of the structural and magnetic properties of $\mathrm{PtCo}-\mathrm{CdS}$ hybrid structures in comparison to similar free-standing PtCo nanoparticles. X-ray absorption spectroscopy (XAS) is utilized as a sensitive probe for identifying subtle differences in the local structure and composition of the hybrid materials, which can be difficult to discern using traditional analytical methods such as electron microscopy and X-ray diffraction (XRD). While the electronic and atomic structures of noble metal-based bimetallic alloys and free-standing PtCo 
nanoparticles have been actively investigated via XAS for the past decade, ${ }^{29-33}$ the application of XAS to nanoscale heterostructures such as our hybrid materials is relatively new and can be effective in forming a more complete picture of compositional variation within these complex nanostructures. Such differences are important because they may have a significant effect on the electronic and surface properties of hybrid materials, which will ultimately dictate their photocatalytic and magnetic properties.

\section{RESULTS AND DISCUSSION}

Structural and magnetic properties were investigated for two different hybrid nanostructures composed of bimetallic PtCo nanoparticles grown selectively on the tips of CdS nanorods and compared to similar free-standing PtCo nanoparticles. Hybrid $\mathrm{PtCo}-\mathrm{CdS}$ samples with different ratios of $\mathrm{Pt} / \mathrm{Co}$ were synthesized using a method described previously, ${ }^{24}$ and a sample of free-standing PtCo nanoparticles containing approximately $15 \%$ Co was prepared according to the method of Shevchenko et al. ${ }^{2}$ The hybrid samples with a 3:1 Pt/Co ratio and a 1:1 Pt/Co ratio, as measured by selected area energy-dispersive $\mathrm{X}$-ray spectroscopy (EDX) on single tips, are referred to as "Low Co" and "High Co", respectively. Figure 1 shows representative transmission electron microscopy (TEM) and highresolution TEM (HRTEM, inset) images of the freestanding PtCo nanoparticles (Figure 1A), Low Co (Figure 1B), and High Co (Figure 1C) hybrid samples. Both the PtCo nanoparticles and the CdS nanorods are crystalline, and the average dimensions of the $C d S$ rods are $120 \times 4 \mathrm{~nm}$. The free-standing PtCo nanocrystals are $3.9 \pm 0.5 \mathrm{~nm}$ in diameter, while the High Co hybrids have tips with an average diameter of $7.2 \pm 2.4 \mathrm{~nm}$ and the Low Co hybrids have $3.6 \pm 1.1 \mathrm{~nm}$ tips. No visible phase separation was observed in the nanoparticle tips of the hybrid structures as a result of the growth process on the CdS rods.

Investigation of the PtCo-CdS hybrid materials by synchrotron-based XRD suggests that growth of PtCo on CdS rods may not necessarily be analogous to the formation of free-standing PtCo nanoparticles in solution. Figure 2 displays diffraction patterns for both hybrid structures, as well as the free-standing nanocrystals. The XRD pattern of the free-standing PtCo nanocrystals exhibits reflections characteristic of a facecentered cubic (fcc) lattice shifted to slightly higher angles $\left(+0.1^{\circ}\right)$ than those expected for bulk Pt, which is consistent with a change in lattice constant due to alloy formation. ${ }^{2,25}$ The peaks observed at 16 and $24^{\circ}$ are artifacts of the XRF tape used in data acquisition. Selected area energy-dispersive X-ray spectroscopy (EDX) analysis performed on single free-standing particles gives an average of $15 \%$ (atomic) Co incorporation relative to $\mathrm{Pt}$, while the change in lattice constant by XRD corresponds to about 5\% Co incorporation. On the
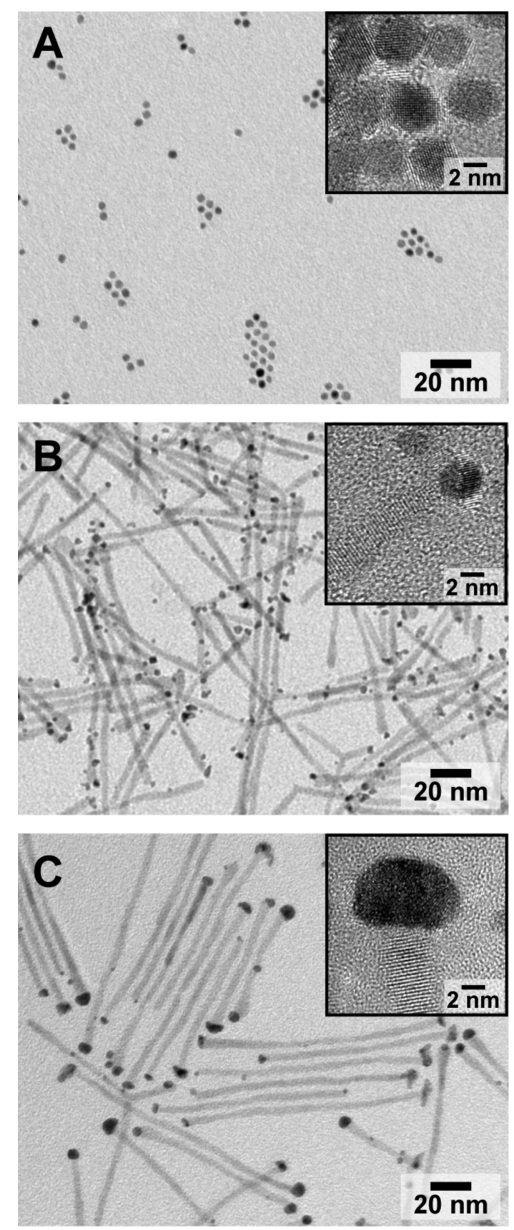

Figure 1. TEM and HRTEM (inset) images of (A) free-standing PtCo nanocrystals $(3.9 \pm 0.5 \mathrm{~nm})$ containing $15 \% \mathrm{Co}$, as determined by selected area EDX, (B) Low Co hybrid nanostructures composed of PtCo nanocrystals ( $3.6 \pm 1.1 \mathrm{~nm}, 25 \%$ Co by EDX) grown on CdS nanorods, and (C) High Co hybrid structures with PtCo tips (7.2 $\pm 2.4 \mathrm{~nm}, 50 \%$ Co by EDX) on CdS rods.

other hand, the XRD patterns for the Low Co and High Co hybrid structures show the presence of crystalline wurtzite CdS and pure Pt, with no peak shifts observed to indicate alloy formation. While both the (111) and (200) reflections of fcc Co overlap with CdS peaks, no other crystalline structures of Co or Co-oxides can be clearly identified in either of the XRD patterns.

Experimentally, we have observed that our Co precursor (cobalt acetate, $\mathrm{Co}(\mathrm{OAc})_{2}$ ) is not reduced in the absence of the Pt precursor under the reaction conditions described here, either with or without the CdS nanorods (see Methods section). These results suggest that either a bimetallic alloy or a Pt-Co core-shell structure is produced on the CdS nanorod tips since Co is found to be present by selected area EDX analysis. However, the HRTEM images in Figure 1 do not show a visible core-shell interface, nor are there shifts in the XRD patterns that are expected for incorporation of Co into a Pt lattice. Although Co-rich nuclei have been proposed for $\mathrm{Pt}_{3} \mathrm{Co}$ alloys formed by the co-reduction of highly reactive $\mathrm{CO}_{2}(\mathrm{CO})_{8}$ with Pt- 


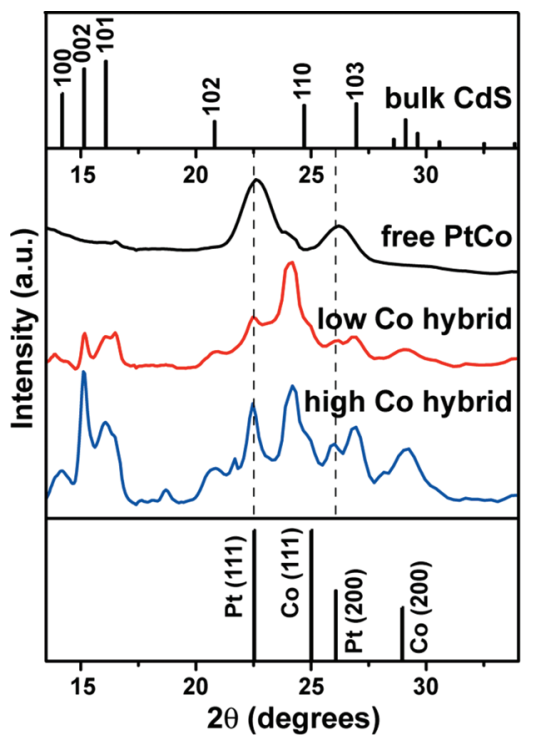

Figure 2. X-ray diffraction patterns for the free-standing PtCo nanocrystals and the Low Co and High Co hybrid nanostructures. The PtCo nanocrystals show a slight Bragg shift to higher angles corresponding to a decrease in lattice constant with Co incorporation, while the hybrid structures do not. The incident X-ray wavelength was approximately $0.88 \AA$.

acetylacetonate $\left(\mathrm{Pt}(\mathrm{acac})_{2}\right){ }_{1}{ }^{26}$ the $\mathrm{Co}(\mathrm{OAc})_{2}$ used here cannot be reduced without the Pt precursor. Pure Pttipped CdS rods, on the other hand, can be easily formed, ${ }^{24}$ suggesting that the formation of PtCo nanoparticles on the tips of the rods may first require the nucleation of Pt metal. For metal combinations having different reduction potentials, the metal with the most favorable potential will often be reduced first. ${ }^{6}$ In the case of NiPt nanocrystals prepared by Weller and coworkers, increasing the amount of $\mathrm{Ni}(\mathrm{OAc})_{2}$ added prior to reaction favors growth rather than nucleation, indicating that Pt-rich nuclei are formed at the outset of reaction. ${ }^{27}$ Therefore, the growth of bimetallic nanoparticles in our hybrid structures may be initiated by nucleation of Pt on the tips of the CdS rod, not only as a consequence of the high surface energy at the tips and the decreased activation energy for heterogeneous nucleation but also due to the high affinity between Pt and $\mathrm{S}$. As growth continues, favorable free energy of mixing promotes incorporation of Co into the metal lattice to form an alloy. The lack of shift in the Bragg peaks predicted by Vegard's law may not necessarily preclude the presence of a PtCo alloy but may be a consequence of strain induced by the growth of the alloy on a Pt nucleate immobilized on a CdS nanorod.

Experimental control samples were prepared in the absence of CdS nanorods to gain a better understanding of how the nanorod substrate influences the binary metal nanoparticle structure. The co-reduction of $\mathrm{Co}(\mathrm{OAc})_{2}$ and $\mathrm{Pt}(\mathrm{acac})_{2}$ was performed in the absence of $\mathrm{CdS}$ nanorods (see Supporting Information, Figure S1A). The resulting nanoparticles contain approximately $40 \%$ Co by bulk EDX analysis, but the XRD shift indicates that only about 7\% Co is incorporated. However, if the same experiment is conducted in the presence of preformed Pt seeds (see Supporting Information, Figure $\mathrm{S} 1 \mathrm{~B}, \mathrm{C})$, the XRD shift is almost nonexistent and yet Co incorporation is still as high as $40 \%$ by bulk EDX analysis. The presence of a Pt seed, whether formed in situ to promote reduction of a less easily reduced metal ${ }^{6}$ or preformed in a separate reaction, may interfere with the lattice spacing of an alloy shell.

If we consider the $\mathrm{Ni}(\mathrm{OAc})_{2}$ precursor, which can be reduced in the absence of $\mathrm{Pt}(\mathrm{acac})_{2}$ under the conditions employed here, the co-reduction of $\mathrm{Ni}(\mathrm{OAc})_{2}$ with $\mathrm{Pt}(\mathrm{acac})_{2}$ gives nanoparticles (see Supporting Information, Figure S1D) that exhibit a significant XRD shift corresponding to $36 \% \mathrm{Ni}$ incorporation. Due to the ease with which $\mathrm{Ni}(\mathrm{OAc})_{2}$ is reduced, a Pt seed should not be necessary for the growth of a PtNi alloy. However, in order to reduce $\mathrm{Ni}(\mathrm{OAc})_{2}$ on $\mathrm{CdS}$ rods, $\mathrm{Pt}(\mathrm{acac})_{2}$ is required; otherwise, Ni nucleates homogeneously in solution rather than on the rod tips. The addition of $\mathrm{Pt}$ to the reaction between $\mathrm{Ni}(\mathrm{OAc})_{2}$ and the $\mathrm{CdS}$ rods to form the PtNi-CdS hybrid, published previously, ${ }^{24}$ also prevents a shift in the diffraction pattern, indicating that a Pt seed particle or Pt sticking layer associated with the $\mathrm{CdS}$ rod may inhibit the expected lattice shift.

In the absence of perfect lattice matching, the growth of one substance onto another can lead to local strain at the interface. Growth of PtCo on a Pt nucleate introduces substrate-induced strain as a result of the lattice mismatch. ${ }^{28}$ Ordinarily, this strain is alleviated as nanoparticle growth proceeds away from the interface, but for very small nanoparticles anchored at the tips of CdS rods, strain relaxation may not occur as readily, leading to a PtCo alloy with a larger lattice parameter.
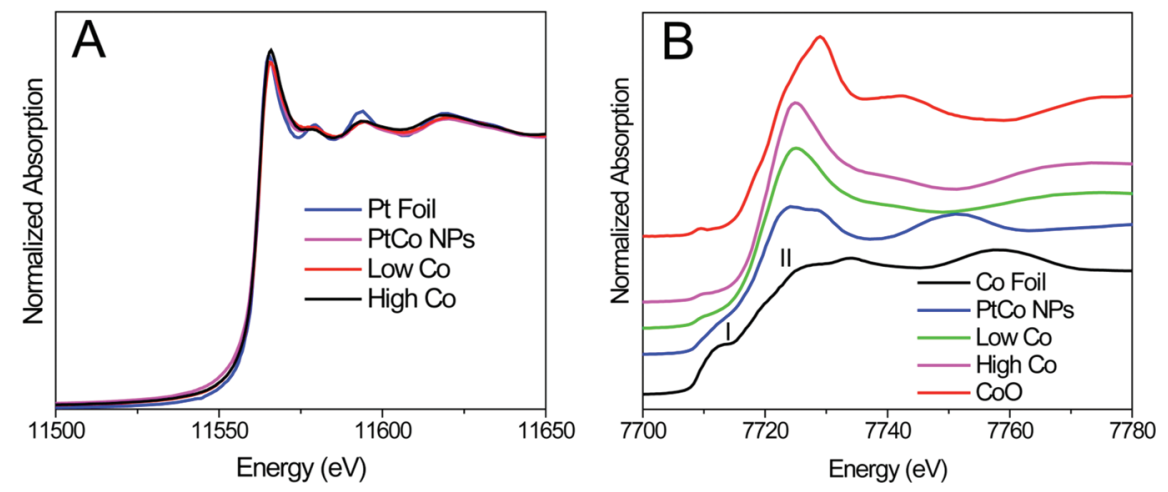

Figure 3. Normalized $\mathrm{Pt}_{\mathrm{L}_{3}-}(\mathrm{A})$ and Co K- (B) edge XANES spectra of free-standing PtCo nanocrystals, Low Co and High Co hybrid nanostructures along with appropriate reference materials. The spectra in (B) are vertically offset for clarity. Feature I is characteristic of metallic Co species, while Feature II is characteristic of $\mathrm{Co}^{2+}$ species. 

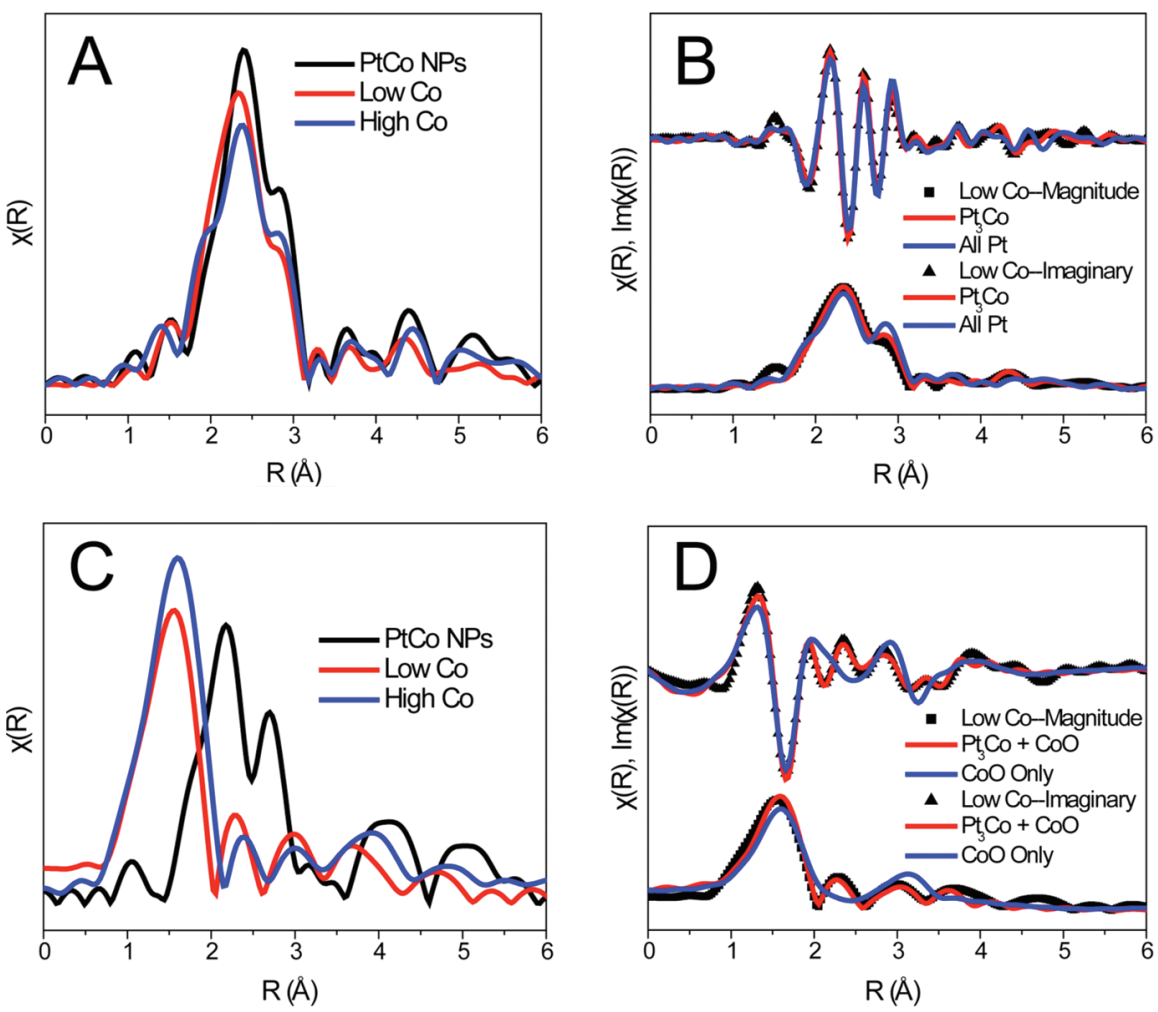

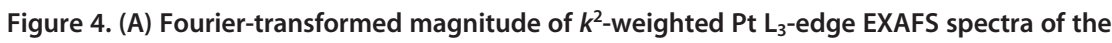
free-standing PtCo nanoparticles and the two PtCo-CdS hybrids. The true interatomic distance is approximately $0.5 \AA$ greater than that indicated by the $x$-axis due to the EXAFS phase shift. (B) Comparison between experimental and theoretical EXAFS spectra for the Low Co hybrid sample with two different model lattices: "All Pt" (fcc Pt structure) and " $\mathrm{Pt}_{3} \mathrm{Co}$ " (fcc Pt structure with $25 \%$ of the $\mathrm{Pt}$ atoms replaced with $\mathrm{Co}$ atoms). The FT magnitude and FT imaginary parts are vertically offset for clarity. (C,D) Similar EXAFS spectra acquired at the Co K-edge. (D) Two theoretical EXAFS spectra employ a rocksalt $\mathrm{CoO}$ structure alone, and a combination of rocksalt $\mathrm{CoO}$ and fcc $\mathrm{Pt}_{3} \mathrm{Co}$.

To gain more insight into the local composition and atomic arrangement of the hybrid nanostructures, X-ray absorption (XAS) measurements were performed, which can provide useful information about bimetallic species, such as details regarding bonding and oxidation state. ${ }^{29}$ Cobalt $\mathrm{K}$ - and $\mathrm{Pt}_{\mathrm{L}}$-edge $\mathrm{X}$-ray absorption near-edge structure (XANES) and extended X-ray absorption fine structure (EXAFS) measurements were carried out on all three samples as well as relevant standard materials. The $\mathrm{Pt}_{3}$-edge XANES spectra (Figure 3A) show that each of the samples (free-standing PtCo nanoparticles and both hybrids) corresponds well with elemental Pt and exhibits little variation or chemical shift across samples in terms of position and amplitude of the main absorption edge peak, also known as the white line. The consistency in these spectra indicates that the Pt in our samples is predominantly metallic and not oxidized in any appreciable way. At the Co K-edge, however, the situation is quite different (Figure $3 \mathrm{~B}$ ). The spectra for the PtCo-CdS hybrids show a decrease in intensity of the shoulder at $7712 \mathrm{eV}$ (Feature I), which indicates a decrease in metallic Co species in the hybrid nanostructures relative to the free-standing PtCo nanocrystals. Additionally, there is a significant increase in the principal absorption edge (white-line) intensity (broad peak at $7725 \mathrm{eV}$, Feature II) for the formation of $\mathrm{Co}^{2+}$ species. Since the XANES spectra represent an average measurement of an ensemble of hybrid materials, the results strongly suggest that the Co atoms reside in at least two distinct environments in the hybrid structures. Features I and II have also been shown to vary drastically upon PtCo alloying ${ }^{30}$ and are observed to differ between the free-standing PtCo nanoparticles and the PtCo-CdS hybrids. These changes reflect a charge transfer from the Co $3 d$ levels to the Pt $5 d$ levels and subsequent population rearrangement in the Co $3 \mathrm{~d}$ states. The presence of the charge-transfer signatures in the Co XANES is consistent with the formation of a bimetallic alloy, as opposed to a core-shell structure. ${ }^{31}$

To further probe the environment of the Pt and Co atoms in these structures, EXAFS measurements were used to determine the local bonding characteristics. This technique relies on the characteristic interference pattern between the outgoing photoelectrons from the absorbing atom and backscattered photoelectrons coming off of neighboring atoms. The oscillatory interference pattern is highly dependent on the identity of the surrounding atoms, as well as the coordination geometry and degree of disorder. By comparing the experimental EXAFS spectra with theoretical spectra obtained from model lattices, which will be discussed presently, we can ascertain the surrounding environment of the absorbing atom and obtain a detailed picture of the local atomic structure of the system.

The nanoparticle growth process that occurs on the tips of a semiconductor nanorod is significantly different than nanoparticle formation free in solution. Consequently, the hybrid architecture may exhibit subtle differences in the bimetallic composition and atomic arrangement, depending on the $\mathrm{Pt} / \mathrm{Co}$ ratio. The $\mathrm{Pt} \mathrm{L}_{3}{ }^{-}$ edge $k^{2}$-weighted EXAFS spectra (shown in the Supporting Information) were Fourier-transformed to real (R) space in the $k$ range of $2.8-11.2 \AA^{-1}$; the $R$ space spectra are shown in Figure $4 \mathrm{~A}$. As with the Pt XANES measurements, there is very little variation observed in the spectra across the three samples. As a result of the large differences in atomic number and mass between Pt and Co, the presence of Co in the Pt lattice should significantly alter the $\mathrm{X}$-ray backscattering properties and, hence, yield different EXAFS spectra. These differ- 
TABLE 1. Statistical EXAFS Parameters from the Best-Fit Models of Each Sample

\begin{tabular}{|c|c|c|c|c|c|c|}
\hline \multirow[b]{2}{*}{ sample } & \multicolumn{3}{|c|}{ Pt $\mathrm{L}_{3}$-edge } & \multicolumn{3}{|c|}{ Co K-edge } \\
\hline & PtCo NPs & Low Co hybrid & High Co hybrid & PtCo NPs & Low Co hybrid & High Co hybrid \\
\hline$R$ factor ${ }^{a}$ & 0.0049 & 0.0198 & 0.0089 & 0.0117 & 0.0112 & 0.0066 \\
\hline $\mathrm{NN}(\AA)^{a}$ & $2.751 \pm 0.002(\mathrm{Pt})$ & $2.731 \pm 0.018(\mathrm{Pt})$ & $2.739 \pm 0.010(\mathrm{Pt})$ & $2.679 \pm 0.070(\mathrm{Pt})$ & $2.062 \pm 0.063(0)$ & $2.053 \pm 0.072(0)$ \\
\hline & $2.660 \pm 0.089\left(C_{0}\right)$ & $2.622 \pm 0.127\left(C_{0}\right)$ & $2.633 \pm 0.116(\mathrm{C} 0)$ & $2.650 \pm 0.099(\mathrm{C} 0)$ & $2.643 \pm 0.106(\mathrm{Co} / \mathrm{Pt})^{b}$ & $2.653 \pm 0.097(\mathrm{Co} / \mathrm{Pt})^{b}$ \\
\hline$\sigma^{2}\left(10^{-3} \AA^{2}\right)^{a}$ & $5.48 \pm 0.86(\mathrm{Pt})$ & $8.34 \pm 1.29(\mathrm{Pt})$ & $6.96 \pm 1.09(\mathrm{Pt})$ & $9.02 \pm 2.58(\mathrm{Pt})$ & $8.60 \pm 5.98(0)$ & $8.15 \pm 5.30(0)$ \\
\hline & $4.62 \pm 2.18(\mathrm{C} 0)$ & $5.63 \pm 2.62(\mathrm{C} 0)$ & $11.60 \pm 7.37(\mathrm{C} 0)$ & $6.40 \pm 3.93(\mathrm{Co})$ & $8.98 \pm 7.09(\mathrm{Co} / \mathrm{Pt})^{b}$ & $4.22 \pm 2.64(\mathrm{Co} / \mathrm{Pt})^{b}$ \\
\hline
\end{tabular}

${ }^{a} R$ factor is the fractional misfit between theory and experiment; NN is nearest-neighbor distance between the absorbing atom and the scattering atom indicated in parentheses, and $\sigma^{2}$ is the mean-square displacement of the scattering atom. ${ }^{b}$ Due to fitting constraints from using two models simultaneously, the interatomic distance between the absorbing $\mathrm{C} 0$ atom and a Co or Pt atom in the bimetallic alloy could not be distinguished in these samples.

ences can be examined by creating model lattices and comparing theoretical EXAFS fits with the experimental EXAFS data. Two model lattices were created for fitting the Pt EXAFS data. The first is a standard fcc Pt lattice; this model lattice is called "All Pt". The second model lattice has a percentage of the Pt atoms in the fcc Pt lattice randomly replaced with Co atoms, in accordance with the EDX data obtained for each sample. For the Low Co hybrid sample, $25 \%$ of the Pt atoms are replaced by Co atoms. This model lattice is called "Alloy".

In the case of the Low Co hybrid sample, as well as with the free-standing PtCo nanoparticles, the "Alloy" model provides the best fit to the data, as shown in Figure 4B. Using the "All Pt" model lattice to fit the experimental Pt EXAFS data from these two samples results in fits that have worse fractional misfits ( $R$ factors) by a factor of 5-10 (for full details, please see the Supporting Information). This suggests that, in spite of the lack of a shift in the XRD Bragg peaks, Co is indeed incorporated into the Pt lattice during nanoparticle growth, forming a bimetallic alloy on the CdS nanorod tips. The Co nearest-neighbor distances (calculated from the "Alloy" model lattice) tend to be slightly smaller than the corresponding Pt distances (Table 1); however, the Co distances also tend to have larger standard deviations, which is suggestive of a greater degree of disorder in the PtCo lattice of the hybrid structures. Thus, while the XRD measurements may reflect an overall average lattice constant that is similar to pure Pt, the EXAFS results suggest there may be some differences in the positions of the Co atoms in the PtCo lattice as a result of the nonisotropic strain induced by a Pt seed on a CdS nanorod substrate. These observations are also reflected in the $k^{2}$-weighted EXAFS spectra at the Co K-edge (see Supporting Information), which shows a high degree of oscillation damping in the hybrid samples that is absent in the free PtCo nanoparticles. For the High Co hybrid sample, on the other hand, the best fractional misfit is obtained when the two model lattices ("All Pt" and "Alloy") are fit simultaneously in a 65:35 amplitude ratio ("Alloy"/"All Pt"). This suggests that the Pt environment is perhaps more complex in this sample, possibly as a result of the larger metal particles present on the nanorod tips.
Although the differences in the Pt EXAFS spectra for the three samples are minimal, the Co K-edge EXAFS data displayed in Figure $4 \mathrm{C}$ indicate that there are significant changes in the environment of the Co atoms. The free-standing nanoparticles can once again be wellmodeled with the fcc PtCo "Alloy" model lattice, but the hybrid structures do not fit this model lattice at all. The most striking observation is the appearance of a new peak at $1.5 \AA$ in the Fourier transformed spectra of the hybrid structures (Figure $4 C$ ), which corresponds to a nearest-neighbor distance of about $2.0 \AA$ (the true interatomic distances are approximately $0.5 \AA$ greater than that indicated from the ordinate of Figure $4 A, C$, due to the EXAFS phase shift). This distance matches very well with the nearest-neighbor distance in the rocksalt structure of $\mathrm{CoO}$. When the hybrid Co EXAFS spectra are fitted using a combination of the fcc PtCo model lattice and a rocksalt $\mathrm{CoO}$ model lattice (Figure $4 D)$, convergence of the fit is obtained with a small mismatch ( $R$ factor $\sim 0.011$; approximately $1 \%$ mismatch). If only one of these two crystal systems is used for the fitting, convergence is not obtained ( $R$ factor $>0.1$ ). Full fitting results for all samples at both metal absorption edges can be found in the Supporting Information.

Coupled with the XANES analysis, the EXAFS measurements suggest that, although the nanoparticles present at the tips of the CdS rods appear homogeneous by TEM, they actually comprise a core of PtCo surrounded by a very thin shell of $\mathrm{CoO}$. These results are in contrast to the data from the free-standing PtCo nanoparticles, which show only the presence of a single, uniform phase. The evidence for a core-shell structure at the tips of the CdS nanorods can be rationalized in the context of the proposed growth mechanism for bimetallic metal nanoparticles on CdS rods. As stated above, it is believed that nanoparticle growth on the CdS tips is initiated by $\mathrm{Pt}$, and not $\mathrm{Co}$, nucleation on the CdS surface. As the particle grows, $\mathrm{Co}$ is incorporated into the Pt lattice, creating a strained bimetallic alloy. Size control of the particle tips is achieved by increasing the amount of metal precursors added to the CdS nanorods at the outset of the reaction. Even though the ratio of $\mathrm{Pt} / \mathrm{Co}$ remains consistent, the amount of Co incorporation measured by EDX spectros- 

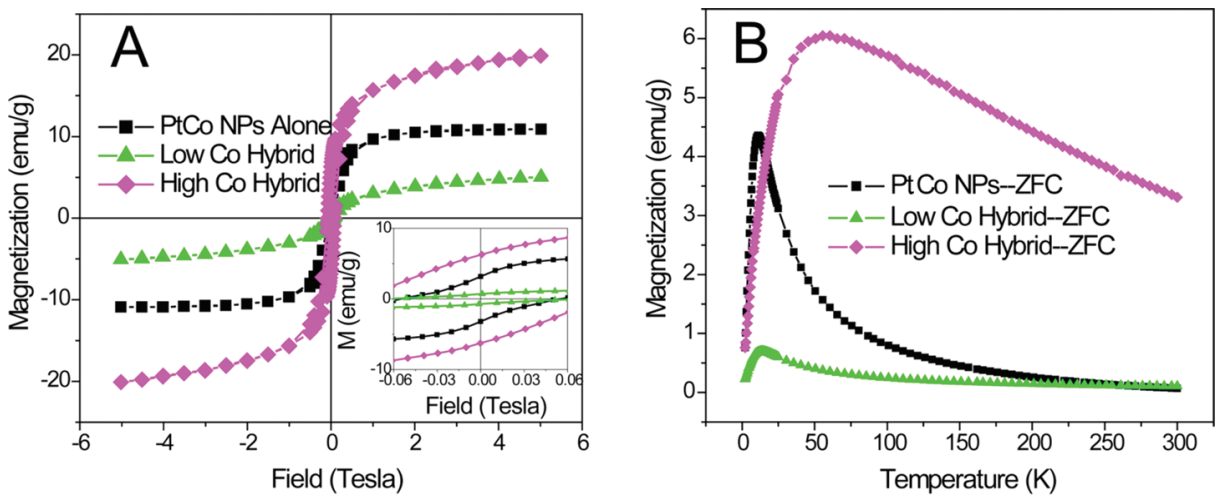

Figure 5. (A) Magnetization vs field measurements at $4.2 \mathrm{~K}$ for both hybrid samples and free-standing PtCo nanoparticles. The magnetization of each sample is normalized to its total mass. (B) Temperature-dependent magnetization measurements obtained by cooling the samples in zerofield and then measuring in an applied field of 1000 Gauss.

copy increases with the nanoparticle tip size. The presence of a Co shell at the nanoparticle surface may be a result of enrichment with Co as growth proceeds away from the strained interface and/or segregation of Co to the surface in an effort to alleviate strain in the particle. ${ }^{6}$ The Co shell could then undergo postsynthetic oxidation upon exposure to air during the magnetic and spectroscopic measurements, resulting in the formation of $\mathrm{CoO}$ as suggested by the EXAFS data. In another bimetallic system, calculations performed for $\mathrm{Ru}_{31} \mathrm{Pt}_{6}$ clusters adsorbed on carbon predict migration of Pt atoms to the cluster surface as a result of the stronger binding of Ru to the carbon substrate which acts as a driving force for segregation. ${ }^{32}$

It should be noted that the predicted $\mathrm{CoO}$ shell is likely to be extremely thin. For example, the average particle diameter in the High Co hybrid sample is approximately $7.2 \mathrm{~nm}$, and the $\mathrm{Pt} / \mathrm{Co}$ elemental ratio, as determined by EDX, is approximately 1:1. On the basis of calculations from the elemental ratios, total particle size, and unit cell volumes of both the PtCo and $\mathrm{CoO}$ phases, we find that the PtCo core diameter would only have to be reduced to about $5.4 \mathrm{~nm}$, leaving a $\mathrm{CoO}$ shell with a thickness of less than $1 \mathrm{~nm}$, in order to satisfy the elemental ratios obtained by EDX spectroscopy. A shell this thin, and possibly amorphous, would be very difficult to detect by TEM or XRD, even at high resolution. The Low Co hybrid sample has smaller particles on the CdS nanorod tips, which would make detection of a $\mathrm{CoO}$ shell on the nanoparticle even more challenging.

It is also worth noting that similar spectroscopic observations have been reported previously for core-shell bimetallic nanoparticles..$^{33-35}$ For example, when Pt nanoparticles are intentionally overcoated with a shell of a first-period transition metal (TM $=\mathrm{Co}$, $\mathrm{Cu}, \mathrm{Fe}), \mathrm{XAS}$ analysis reveals a chemical shift of the TM XANES spectrum to a value between that of the pure metal and the divalent oxide, and EXAFS indicates the presence of both $\mathrm{TM}-\mathrm{O}$ bonding and $\mathrm{TM}-\mathrm{Pt}$ bonding. Each effect can be explained by the oxidation of the sur- face TM atoms. Thus, the picture of our system that emerges is that of a core-shell nanoparticle comprising a bimetallic PtCo alloy at the core and a very thin $\mathrm{CoO}$ shell at the surface, with the nanoparticle supported on the tip of a CdS nanorod. In our case, the core-shell geometry is unintentional, and given that we observe its spectral signatures only in the hybrid nanostructures, we believe this geometry arises as a consequence of the differing nanoparticle growth mechanism that occurs on the surface of the semiconductor nanorods as opposed to free nanoparticle growth in solution.

The complex organization of the bimetallic tips in the hybrid structures may have an effect on the magnetic properties of the hybrid structures relative to their unhybridized counterpart. Figure 5 shows magnetic measurements taken on all three samples in powder form. At low temperatures, hysteresis is observed in all three samples when the magnetization is measured as a function of applied field. In the absence of pure Co metal, which we do not observe in our samples, the presence of hysteresis lends additional support for the incorporation of Co into the Pt lattice, since, in the bulk, neither pure Pt nor $\mathrm{CoO}$ possesses ferromagnetic properties. Temperature-dependent measurements (Figure $5 \mathrm{~B}$ ) indicate that both the free-standing nanoparticles and the hybrid structures exhibit superparamagnetic behavior, which is typical of nanoscale PtCo particles. ${ }^{3}$ The blocking temperature, $T_{\mathrm{B}}$, where the zero-fieldcooled (ZFC) and field-cooled (FC) measurements diverge, increases slightly from the free nanoparticles to the Low Co sample, while a far greater increase is observed for the High Co hybrid, which is commensurate with a larger particle size on the tips of the CdS rods. The peak magnetization in the ZFC measurement for the High Co hybrid sample extends over a wider temperature range than that for the Low Co hybrid or the free-standing PtCo nanoparticles as a result of the larger size distribution of the PtCo tips.

Although the growth of PtCo on CdS nanorods promotes the formation of a more complex bimetallic structure, the magnetic properties of the nanoparticles are retained leading to a multifunctional hybrid material. Additionally, the hybrid architecture of a ferromagnetic metal in contact with a semiconductor is very similar to that employed in studies of spin injection. ${ }^{36-38}$ It has been shown that both paramagnetic materials as well as dilute magnetic semiconducting materials can be endowed with spin order near the interface of a ferromagnetic ma- 
terial. It is possible that the presence of ferromagnetic bimetallic nanoparticles grown on nanorod tips can align a fraction of the free carriers in the CdS rods. Such hybrid structures, with the ferromagnetic component grown directly onto the semiconductor, could be useful for studying nanoscale spin injection into semiconductor nanostructures.

\section{CONCLUSION}

The analysis presented here is a clear demonstration of the new and unique properties resulting from the creation of a hybrid nanostructure. With the direct growth of PtCo nanoparticles onto the tips of $\mathrm{CdS}$ nanorods, we have observed unexpected structural changes in the composition of the metal nanoparticle tips, as indicated by X-ray absorption spectroscopy measurements. Despite the presence of a cobalt oxide surface layer, the magnetic properties of the alloy particles attached to the $\mathrm{CdS}$ tips are preserved. With the ability to directly grow catalytic or magnetically active metal nanoparticles onto semiconductor nanostructures, we can create hybrid materials that can be precisely tailored for desired applications. At the same time, the utilization of advanced characterization techniques becomes increasingly important for identifying and understanding compositional variation within hybrid nanostructures which will play a significant role in the resulting properties of these materials.

\section{METHODS}

Hybrid PtCo-CdS nanostructures with different ratios of $\mathrm{Pt} / \mathrm{Co}$ were synthesized using a method described previously, ${ }^{24}$ and free-standing PtCo nanoparticles containing 15\% (atomic) Co were prepared according to the method described by Shevchenko et al. ${ }^{2}$ The hybrid samples were thoroughly cleaned prior to analysis to remove any free-standing particles.

TEM analysis was performed on a Tecnai G2 S-Twin microscope operated at $200 \mathrm{kV}$, and HRTEM on a JEOL $2100 \mathrm{~F}$ at 200 kV, or a Philips CM200/FEG microscope (200 kV) equipped with a Link EDS detector for EDX spectroscopy. Suspensions of the nanostructures were dispersed on carbon-coated copper grids for electron microscopy and EDX analysis. The sizes of the nanoparticle tips in the $\mathrm{PtCo}-\mathrm{CdS}$ hybrid structures were measured across the longest axis.

The XRD and XAS measurements were conducted at the hard X-ray microprobe beamline 10.3.2 of the Advanced Light Source at the Lawrence Berkeley National Laboratory. ${ }^{39}$ Diffraction data were recorded in transmission mode with a Bruker SMART6000 CCD camera at $14 \mathrm{keV}(\lambda \sim 0.886 \AA)$ incident energy, with a beam size of $16 \times 7 \mu \mathrm{m}^{2}$ and exposure time of $240 \mathrm{~s}$. The two-dimensional diffraction patterns were radially integrated to obtain XRD profiles and calibrated, using the Fit2D software and an alumina $\left(\alpha-\mathrm{Al}_{2} \mathrm{O}_{3}\right)$ standard. Bragg reflections observed in the XRD spectra at approximately 16 and $24^{\circ}$ originate from the XRF tape on which the samples were mounted. XAS spectra were acquired with a Si(111) crystal monochromator, in both transmission and fluorescence modes. The fluorescence and transmission yields were measured with a 7-element Ge solid-state detector (Canberra) and an ion chamber filled with pure $\mathrm{N}_{2}$, respectively. Energies were calibrated using $\mathrm{Co}$ and $\mathrm{Pt}$ metal foils, with first inflection point at 7708.78 and $11562.76 \mathrm{eV}$, respectively. ${ }^{40}$ All samples were measured at room temperature.

The spectra were calibrated, averaged, pre-edge background subtracted, and postedge normalized using standard procedures and custom LabView based software available at the beamline. The $k^{2}$-weighted EXAFS spectra were obtained in various wave vector $(k)$ ranges: $2.8-11.2 \AA^{-1}$ for the Pt edge (all three samples), $2.2-8.4 \AA^{-1}$ for the free-standing PtCo nanoparticles at the Co edge, and $2.2-8.4 \AA^{-1}$ for the PtCo-CdS hybrid samples at the Co edge. The spectra were Fourier-transformed to real $(R)$ space using a Kaiser-Bessel window and fitted with the FEFF8.0 program using the software ARTEMIS. Theoretical scattering paths were obtained from crystallographic descriptions of known models, and structural parameters, such as the coordination number, the interatomic distance, and the Debye-Waller factor, were obtained from the fitting. Full information on the EXAFS analysis can be found in the Supporting Information.

For magnetic measurements, the samples were dried as films and their magnetic properties measured with a SQUID magnetometer (Quantum Design MPMS XL). Field-dependent $(M-H)$ magnetization measurements were conducted at $4.2 \mathrm{~K}$, while temperature-dependent $(M-T)$ measurements were conducted from 2 to $300 \mathrm{~K}$ with an applied field of 1000 Gauss after cooling the sample to $2 \mathrm{~K}$ in zero-field.

Acknowledgment. We thank J. Long for the use of the SQUID magnetometer, P. Alivisatos for the use of the TEM, and P. Yang and M. Marcus for helpful discussion. We also thank the National Center for Electron Microscopy at the Lawrence Berkeley National Laboratory for the use of their microscope facilities. Work at the Molecular Foundry was supported by the Director, Office of Science, Office of Basic Energy Sciences, Division of Materials Sciences and Engineering, U.S. Department of Energy, under Contract DE-AC02-05CH11231.

Supporting Information Available: TEM images of the metal nanoparticle control samples, full details for EXAFS data processing as well as the $k$-space EXAFS spectra, details regarding the construction of the model lattices with cartoon representations, and fitting results for the EXAFS spectra. Room-temperature field-dependent magnetization measurements can also be found. This material is available free of charge via the Internet at http://pubs.acs.org.

\section{REFERENCES AND NOTES}

1. Sun, S.; Murray, C. B.; Weller, D.; Folks, L.; Moser, A. Monodisperse FePt Nanoparticles and Ferromagnetic FePt Nanocrystal Superlattices. Science 2000, 287, 1989-1992.

2. Shevchenko, E. V.; Talapin, D. V.; Rogach, A. L.; Kornowski, A.; Haase, M.; Weller, H. Colloidal Synthesis and SelfAssembly of $\mathrm{CoPt}_{3}$ Nanocrystals. J. Am. Chem. Soc. 2002 , 124, 11480-11485.

3. Wiekhorst, F.; Shevchenko, E.; Weller, H.; Kötzler, J. Anisotropic Superparamagnetism of Monodispersive Cobalt-Platinum Nanocrystals. Phys. Rev. B 2003, 67, 224416.

4. Jürgens, B.; Borchert, H.; Ahrenstorf, K.; Sonström, P.; Pretorius, A.; Schowalter, M.; Gries, K.; Zielasek, V.; Rosenauer, A.; Weller, H.; et al. Colloidally Prepared Nanoparticles for the Synthesis of Structurally WellDefined and Highly Active Heterogeneous Catalysts. Angew. Chem., Int. Ed. 2008, 47, 8946-8949.

5. Alayoglu, S.; Nilekar, A. U.; Mavrikakis, M.; Eichhorn, B. RuPt Core-Shell Nanoparticles for Preferential Oxidation of Carbon Monoxide in Hydrogen. Nat. Mater. 2008, 7, 333-338.

6. Ferrando, R.; Jellinek, J.; Johnston, R. L. Nanoalloys: From Theory to Applications of Alloy Clusters and Nanoparticles. Chem. Rev. 2008, 108, 845-910.

7. Stamenkovic, V. R.; Fowler, B.; Mun, B. S.; Wang, G.; Ross, P. N.; Lucas, C. A.; Marković, N. M. Improved Oxygen Reduction Activity on $\mathrm{Pt}_{3} \mathrm{Ni}(111)$ via Increased Surface Site Availability. Science 2007, 315, 493-497. 
8. Stamenkovic, V. R.; Mun, B. S.; Arenz, M.; Mayrhofer, K. J. J.; Lucas, C. A.; Wang, G.; Ross, P. N.; Marković, N. M. Trends in Electrocatalysis on Extended and Nanoscale PtBimetallic Alloy Surfaces. Nat. Mater. 2007, 6, 241-247.

9. Tao, F.; Grass, M. E.; Zhang, Y.; Butcher, D. R.; Renzas, J. R.; Liu, Z.; Chung, J. Y.; Mun, B. S.; Salmeron, M.; Somorjai, $\mathrm{G}$. A. Reaction-Driven Restructuring of $\mathrm{Rh}-\mathrm{Pd}$ and $\mathrm{Pt}-\mathrm{Pd}$ Core-Shell Nanoparticles. Science 2008, 322, 932-934.

10. Cozzoli, P. D.; Pellegrino, T.; Manna, L. Synthesis, Properties and Perspectives of Hybrid Nanocrystal Structures. Chem. Soc. Rev. 2006, 35, 1195-1208.

11. Mokari, T.; Rothenberg, E.; Popov, I.; Costi, R.; Banin, U. Selective Growth of Metal Tips onto Semiconductor Quantum Rods and Tetrapods. Science 2004, 304, 1787-1790.

12. Mokari, T.; Sztrum, C. G.; Salant, A.; Rabani, E.; Banin, U. Formation of Asymmetric One-Sided Metal-Tipped Semiconductor Nanocrystal Dots and Rods. Nat. Mater. 2005, 4, 855-863.

13. Salant, A.; Amitay-Sadovsky, E.; Banin, U. Directed SelfAssembly of Gold-Tipped CdSe Nanorods. J. Am. Chem. Soc. 2006, 128, 10006-10007.

14. Choi, J.-S.; Jun, Y. w.; Yeon, S. I.; Kim, H. C.; Shin, J. S.; Cheon, J. Biocompatible Heterostructured Nanoparticles for Multimodal Biological Detection. J. Am. Chem. Soc. 2006, 128, 15982-15983.

15. Pellegrino, T.; Fiore, A.; Carlino, E.; Giannini, C.; Cozzoli, P. D.; Ciccarella, G.; Respaud, M.; Palmirotta, L.; Cingolani, R.; Manna, L. Heterodimers Based on $\mathrm{CoPt}_{3}-\mathrm{Au}$ Nanocrystals with Tunable Domain Size. J. Am. Chem. Soc. 2006, 128, 6690-6698.

16. Bao, N.; Shen, L.; Takata, T.; Domen, K. Self-Templated Synthesis of Nanoporous CdS Nanostructures for Highly Efficient Photocatalytic Hydrogen Production under Visible Light. Chem. Mater. 2008, 20, 110-117.

17. Tada, H.; Mitsui, T.; Kiyonaga, T.; Akita, T.; Tanaka, K. AllSolid-State Z-Scheme in $\mathrm{CdS}-\mathrm{Au}-\mathrm{TiO}_{2}$ Three-Component Nanojunction System. Nat. Mater. 2006, 5, 782-786.

18. Kamat, P. V.; Flumiani, M.; Dawson, A. Metal-Metal and Metal-Semiconductor Composite Nanoclusters. Colloids Surf., A 2002, 202, 269-279.

19. Subramanian, V.; Wolf, E. E.; Kamat, P. V. Green Emission to Probe Photoinduced Charging Events in $\mathrm{ZnO}-\mathrm{Au}$ Nanoparticles. Charge Distribution and Fermi-Level Equilibration. J. Phys. Chem. B 2003, 107, 7479-7485.

20. Hirakawa, T.; Kamat, P. V. Charge Separation and Catalytic Activity of Ag@TiO 2 Core-Shell Composite Clusters under UV Irradiation. J. Am. Chem. Soc. 2005, 127, 3928-3934.

21. Cozzoli, P. D.; Curri, M. L.; Agostiano, A. Efficient Charge Storage in Photoexcited $\mathrm{TiO}_{2}$ Nanorod-Noble Meta Nanoparticle Composite Systems. Chem. Commun. 2005, 3186-3188.

22. Costi, R.; Saunders, A. E.; Elmalem, E.; Salant, A.; Banin, U. Visible Light-Induced Charge Retention and Photocatalysis with Hybrid CdSe-Au Nanodumbbells. Nano Lett. 2008 8, 637-641.

23. Elmalem, E.; Saunders, A. E.; Costi, R.; Salant, A.; Banin, U. Growth of Photocatalytic CdSe-Pt Nanorods and Nanonets. Adv. Mater. 2008, 20, 4312-4317.

24. Habas, S. E.; Yang, P.; Mokari, T. Selective Growth of Metal and Binary Metal Tips on CdS Nanorods. J. Am. Chem. Soc. 2008, 130, 3294-3295.

25. Ahrenstorf, K.; Albrecht, O.; Heller, H.; Kornowski, A.; Görlitz, D.; Weller, H. Colloidal Synthesis of $\mathrm{Ni}_{x} \mathrm{Pt}_{1-x}$ Nanoparticles with Tuneable Composition and Size. Small 2007, 3, 271-274.

26. Shevchenko, E. V.; Talapin, D. V.; Schnablegger, $H_{\text {.; }}$ Kornowski, A.; Festin, O.; Svedlindh, P.; Haase, M.; Weller, H. Study of Nucleation and Growth in the Organometallic Synthesis of Magnetic Alloy Nanocrystals: The Role of

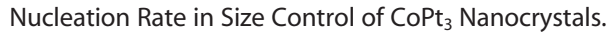
J. Am. Chem. Soc. 2003, 125, 9090-9101.

27. Ahrenstorf, K.; Heller, H.; Kornowski, A.; Broekaert, J. A. C.; Weller, $\mathrm{H}$. Nucleation and Growth Mechanism of $\mathrm{Ni}_{x} \mathrm{Pt}_{1-x}$ Nanoparticles. Adv. Funct. Mater. 2008, 18, 3850-3856.
28. Kim, M.-G.; Shin, S.-C. Temperature Dependence of Growth Morphology of Sputtered (FePt/Pt) Films on MgO (100) Substrate. J. Appl. Phys. 2001, 90, 2211-2215.

29. Hwang, B.-J.; Sarma, L. S.; Chen, C.-H.; Bock, C.; Lai, F.-J.; Chang, S.-H.; Yen, S.-C.; Liu, D.-G.; Sheu, H.-S.; Lee, J.-F. Controlled Synthesis and Characterization of $\mathrm{Ru}_{\text {core }}-\mathrm{Pt}_{\text {shell }}$ Bimetallic Nanoparticles. J. Phys. Chem. C 2008, 112, 19922-19929.

30. Hlil, E. K.; Baudoing-Savois, R.; Moraweck, B.; Renouprez, A. J. X-ray Absorption Edges in Platinum-Based Alloys. 2. Influence of Ordering and of the Nature of the Second Metal. J. Phys. Chem. 1996, 100, 3102-3107.

31. Park, J.-I.; Kim, M. G.; Jun, Y.; Lee, J. S.; Lee, W.; Cheon, J. Characterization of Superparamagnetic "Core-Shell" Nanoparticles and Monitoring Their Anisotropic Phase Transition to Ferromagnetic "Solid Solution" Nanoalloys. J. Am. Chem. Soc. 2004, 126, 9072-9078.

32. Wang, L.-L.; Khare, S. V.; Chirita, V.; Johnson, D. D.; Rockett, A. A.; Frenkel, A. I.; Mack, N. H.; Nuzzo, R. G. Origin of Bulklike Structure and Bond Length Disorder of $\mathrm{Pt}_{37}$ and $\mathrm{Pt}_{6} \mathrm{Ru}_{31}$ Clusters on Carbon: Comparison of Theory and Experiment. J. Am. Chem. Soc. 2006, 128, 131-142.

33. Girardon, J.-S.; Quineta, E.; Griboval-Constant, A.; Chernavskii, P. A.; Gengembre, L.; Khodakov, A. Y. Cobalt Dispersion, Reducibility, and Surface Sites in Promoted Silica-Supported Fischer-Tropsch Catalysts. J. Catal. 2007, 248, 143-157.

34. Hwang, B. J.; Tsai, Y. W.; Sarma, L. S.; Chen, C. H.; Lee, J. F.; Strehblow, H. H. In Situ XAS Investigation of Transformation of Co Monolayer on Carbon-Supported Platinum Clusters Underpotential Control. J. Phys. Chem. B 2004, 108, 15096-15102.

35. Wang, D.-Y.; Chen, C.-H.; Yen, H.-C.; Lin, Y.-L.; Huang, P.-Y.; Hwang, B.-J.; Chen, C.-C. Chemical Transformation from FePt to $\mathrm{Fe}_{1-x} \mathrm{PtM}_{x}(\mathrm{M}=\mathrm{Ru}, \mathrm{Ni}, \mathrm{Sn})$ Nanocrystals by a Cation Redox Reaction: X-ray Absorption Spectroscopic Studies. J. Am. Chem. Soc. 2007, 129, 1538-1540.

36. Maccherozzi, F.; Sperl, M.; Panaccione, G.; Minar, J.; Polesya, S.; Ebert, H.; Wurstbauer, U.; Hochstrasser, M.; Rossi, G.; Woltersdorf, G.; et al. Evidence for a Magnetic Proximity Effect up to Room Temperature at Fe/(Ga,Mn)As Interfaces. Phys. Rev. Lett. 2008, 101, 267201.

37. Johnston-Halperin, E.; Lofgreen, D.; Kawakami, R. K.; Young, D. K.; Coldren, L.; Gossard, A. C.; Awschalom, D. D. Spin-Polarized Zener Tunneling in (Ga,Mn)As. Phys. Rev. B 2002, 65, 041306.

38. Unguris, J.; Celotta, R. J.; Pierce, D. T. Magnetism in Cr Thin Films on Fe(100). Phys. Rev. Lett. 1992, 69, 1125.

39. Marcus, M. A.; MacDowell, A. A.; Celestre, R.; Manceau, A Miller, T.; Padmore, H. A.; Sublett, R. E. Beamline 10.3.2 at ALS: A Hard X-ray Microprobe for Environmental and Materials Sciences. J. Synchrotron Radiat. 2004, 11, 239-247.

40. Kraft, S.; Stumpel, J.; Becker, P.; Kuetgens, U. High Resolution X-ray Absorption Spectroscopy with Absolute Energy Calibration for the Determination of Absorption Edge Energies. Rev. Sci. Instrum. 1996, 67, 681-687. 